\title{
Evaluación De La Influencia De Las Condiciones De Fondo De Pozo En El Deterioro De Un Acero API P110, En Ambientes Simulados Del Proceso De Combustión In Situ, Por Gravimetría Y EIS
}

\author{
Evaluation Of The Influence Of The Conditions Of \\ Downhole In The Deterioration Of A API P110 \\ Steel, Simulated Environments In Situ \\ Combustion Process By Means Of \\ Gravimetric And EIS
}

\author{
Jhon E. Torres ${ }^{1}$, Darío Yesid Peña ${ }^{1}$, \\ Dionisio Laverde ${ }^{1}$
}

\footnotetext{
${ }^{1}$ Grupo de investigaciones en corrosión - GIC Universidad Industrial de Santander (UIS), Bucaramanga, Colombia. Centro de materiales y Nanomateriales CMN-UIS

e-mail: jtorram@gmail.com, darioypb@gmail.com, dlavere@uis.edu.co
}

\section{RESUMEN}

Dada la gran cantidad de consumo del petróleo como recurso energético, tiene por consecuencia una diminución en el crudo existente a nivel mundial, la explotación del crudo pesado surge como nueva alternativa de extracción que se deja a un lado debido a las dificultades asociadas a su procesamiento. Por lo tanto el proceso de combustión in situ, es un método de extracción que facilita la producción de este tipo de crudo, pues generan un aumento en la producción a expensas de un aumento de los costos asociados a mantenimiento, pues a las condiciones operacionales se favorecen fenómenos como la corrosión de los diferentes materiales involucrados en el proceso, pues están expuestos a atmosferas de sulfuro de hierro $\left(\mathrm{H}_{2} \mathrm{~S}\right)$, entre otros gases; a temperaturas y presiones elevadas, lo cual promueve la corrosión y los fenómenos de Sulfide stress cracking. Adicionalmente, los materiales se ven afectados por la acidez y la cantidad de impurezas presentes en el crudo. Por lo anterior se evaluó el comportamiento del acero API P110 expuesto a crudo pesado con diferentes contenidos de azufre ( 0.5 - $1.5 \%$ en peso), TAN (2.28 - $6.84 \mathrm{~g} \mathrm{KOH/g} \mathrm{crudo)} \mathrm{a} \mathrm{elevadas} \mathrm{temperaturas} \mathrm{(190}$ $\left.-250^{\circ} \mathrm{C}\right)$ y presiones $\left(8.96 \times 10^{6}-11.72 \times 10^{6} \mathrm{~Pa}\right)$, ya que se pueden presentar estas condiciones en el proceso de extracción por combustión in situ en fondos de pozo. Se encontró que el principal mecanismo de corrosión en estas condiciones es la corrosión por sulfidación cuyos principales productos de corrosión son el FeS y óxido de hierro $\left(\mathrm{Fe}_{3} \mathrm{O}_{4}\right)$.

Palabras clave: Velocidad de corrosión, EIS, gravimetría, sulfidación, alta temperatura y altas presiones.

\section{ABSTRACT}

Given the large amount of consumption of oil as an energy source, is resulting in a diminution in the existing world oil, the exploitation of heavy oil emerges as new alternative extraction is left aside because of the difficulties associated to processing. Therefore the in situ combustion process is an extraction method that facilitates production of such oil therefore lead to increased production at the expense of an increase in costs associated with maintenance, as operational conditions promote corrosion phenomena as the different materials involved in the process, since they are exposed to atmospheres of iron Sulfide $\left(\mathrm{H}_{2} \mathrm{~S}\right)$, among other gases; at elevated temperatures and pressures, which promotes corrosion phenomena Sulfide stress cracking. Additionally, the materials affected by the acidity and the amount of impurities present in the crude. Therefore the behaviour of API P110 steel exposed to heavy oil was evaluated with different sulphur contents (0.5 - 1.5 wt\%), TAN (2.28 - $6.84 \mathrm{~g} \mathrm{KOH} / \mathrm{g}$ oil) at elevated temperatures $\left(190-250{ }^{\circ} \mathrm{C}\right)$ and pressures $\left(8.96 \times 10^{6}\right.$ to $11.72 \times 10^{6} \mathrm{~Pa}$ ), since these conditions may occur in the extraction process by in situ combustion downhole. It was found that the main mechanism of corrosion in these conditions is sulfidation corrosion corrosion whose 
main products are the $\mathrm{FeS}$ and iron oxide $\left(\mathrm{Fe}_{3} \mathrm{O}_{4}\right)$.

Keywords: Corrosion rate, EIS, gravimetry, sulfidation, high temperature and high pressure.

\section{INTRODUCCIÓN}

El método de recobro térmico de combustión in situ no es una técnica muy reciente, pero debido a la gran cantidad de reservas de crudo pesado y al hallazgo de nuevos yacimientos, es un proceso de gran interés para la extracción de crudos pesado. La combustión in situ involucra la inyección de gas oxidante en un pozo petrolero con el fin de aumentar la temperatura del mismo, dadas las reacciones de oxidación y pirólisis que se generan en el fondo del yacimiento. Estas reacciones de oxidación se dan cuando el gas oxidante reacciona con el coque presente en el hidrocarburo formando un frente de combustión, el cual irá avanzando hacia el pozo productor por el incremento de temperatura, disminuyendo la viscosidad del crudo y haciendo más fácil y rápida la extracción del hidrocarburo.

La exposición de los diferentes materiales involucrados en el proceso de extracción a las condiciones de temperatura que se alcanzan, pueden presentar deterioro por efecto de los agentes corrosivos que tiene el crudo, especialmente por la presencia de ácido nafténico y los compuestos orgánicos de azufre. Por lo tanto, es importante entender el comportamiento de la corrosión del acero API P110 en condiciones de procesamiento y extracción, en un proceso de combustión in situ donde se presenta combustión completa (consumo total de oxigeno).

\section{- Corrosión por ácidos nafténicos.}

Los ácidos nafténicos son ácidos orgánicos presentes en los crudos, cuya fórmula química está compuesta por anillos ciclopentano o ciclohexano, acompañado de una cadena alifática que tiene un grupo carboxilo terminal. Este tipo de compuestos presenta una estructura de la forma: $\mathrm{R}\left(\mathrm{CH}_{2}\right) \mathrm{nCOOH}$, donde n es mayor a 12 .

La disolución de un acero por efecto del ácido nafténico, presenta una serie de reacciones de absorción de iones nafténico (NA) en sitios activos de una determinada superficie, seguida por una desorción de la superficie, es decir, una desorción de las especies solubles formadas por las siguientes reacciones [1]:

$$
\begin{aligned}
& \mathrm{Fe}+(\mathrm{NA})^{-}{ }_{\text {(ads.) }} \rightarrow \mathrm{Fe}(\mathrm{NA})_{\text {(ads.) }}+\mathrm{e}^{-} \\
& \mathrm{Fe}(\mathrm{NA})_{\text {(ads.) }}+(\mathrm{NA})^{-} \rightarrow \mathrm{Fe}(\mathrm{NA})_{2 \text { (ads.) }}+\mathrm{e}^{-} \\
& \mathrm{Fe}(\mathrm{NA})_{2 \text { (ads.) }} \rightarrow \mathrm{Fe}(\mathrm{NA})_{2 \text { (aqu.) }}
\end{aligned}
$$

En términos de la formula química de los ácidos nafténicos, las anteriores reacciones se describe de forma global como:

$\mathrm{Fe}+2 \mathrm{R}\left[\mathrm{CH}_{2}\right] \mathrm{nCOOH} \rightarrow \mathrm{Fe}(\mathrm{R}[\mathrm{CH} 2] \mathrm{nCOO})_{2}+\mathrm{H}_{2}$

Donde R representa al radical nafténico (uno o más anillos saturados de ciclopentano y ciclohexano) unido con grupos carboxilos y $\mathrm{CH}_{2}$, los cuales forman productos de corrosión solubles en el crudo.

El efecto de los ácidos nafténicos en la corrosión de los aceros, se presenta a temperaturas entre $220^{\circ} \mathrm{C} \mathrm{y}$ $350^{\circ} \mathrm{C}$. Además, Gheorghe M. Bota, et al., evidenció que la corrosión por ácidos nafténicos incrementa el triple con el aumento de $55^{\circ} \mathrm{C}$ en la temperatura [2].

- Efecto de corrosión por $\mathrm{H}_{2} \mathrm{~S}$

La influencia del $\mathrm{H}_{2} \mathrm{~S}$ en las reacciones catódicas incluyendo la reducción de protones, son las siguientes:

$\mathrm{H}_{2} \mathrm{~S} \rightarrow \mathrm{H}^{+}+\mathrm{HS}^{-}$
$\mathrm{HS}^{-} \rightarrow \mathrm{H}^{+}+\mathrm{S}^{2-}$
$2 \mathrm{H}^{+}+2 \mathrm{e}^{-} \rightarrow \mathrm{H}_{2}$

El mecanismo de corrosión por $\mathrm{H}_{2} \mathrm{~S}$ en aceros al carbono, está determinado por la formación de productos de corrosión, obtenidos de las reacciones entre los iones de hierro y el $\mathrm{H}_{2} \mathrm{~S}$, produciendo películas de 
sulfuro de hierro. La reacción global de la corrosión por $\mathrm{H}_{2} \mathrm{~S}$ es:

$\mathrm{Fe}_{(\mathrm{s})}+\mathrm{H}_{2} \mathrm{~S} \rightarrow \mathrm{FeS}_{(\mathrm{s})}+\mathrm{H}_{2}$

Esta reacción de corrosión da como resultado la formación de sulfuro de hierro sobre la superficie del material y bajo determinadas condiciones, el sulfuro de hierro sirve como material protector disminuyendo la velocidad de corrosión del acero $[3,4,5]$.

\section{MATERIALES Y MÉTODOS}

\subsection{Materiales}

Las probetas de acero API P110, cuya composición química elemental obtenida por espectroscopía de emisión óptica se puede ver en la tabla 1 y es comparada con la Norma NTC-4713 (2012) en donde se aclaran las especificaciones técnicas de este tipo de materiales para la industria del petróleo, se prepararon con dimensiones de 76×13×1.2 mm con dos orificios de $7 \mathrm{~mm}$ de diámetro para sujetar las probetas en la autoclave.

Tabla 1: Composición química de los aceros API P110.

\begin{tabular}{c|c|c|c|c|c|c|c}
\hline MATERIAL & \%C & \%Mn & \%Si & \%Cr & \%Ni & \%P & \%S \\
\hline NTC 4713 & --- & --- & --- & --- & --- & $<0.03$ & $<0.03$ \\
\hline Muestra de trabajo & 0.33 & 1.215 & 0.328 & 0.096 & 0.103 & 0.016 & $<0.15$ \\
\hline
\end{tabular}

Estas probetas fueron desbastadas con lijas de carburo de silicio hasta la lija número 600 y desengrasadas en un baño ultrasónico en acetona por 5 minutos, posteriormente se pesaron en una balanza analítica con un error de \pm 0.0001 . Al finalizar los ensayos, a los cupones se les hizo una limpieza mecánica y en acetona, con el fin de eliminar cualquier rastro de crudo sobre los cupones, para posteriormente realizar el pesaje y decapado de las mismas para determinar la velocidad de corrosión. La solución de decapado para el acero P110 fue una solución de $500 \mathrm{ml}$ de $\mathrm{HCl}$ con 3.5 g de Hexametileno tetramina con $500 \mathrm{ml}$ de agua destilada (norma ASTM G1(2011)) con una duración de decapado de 2 minutos.

\subsection{Caracterización del crudo usado}

El crudo usado para llevar a cabo las pruebas experimentales tenía un porcentaje en peso de azufre total de $1.5 \%$, un numero de acidez total (TAN) de $6.84 \mathrm{Mg} \mathrm{KOH/g}$, un grado API de 12.4 y una densidad de 985 $\mathrm{Kg} / \mathrm{m}^{3}$; y se usó para cada una de las pruebas 1 litro de crudo pesado. Para modificar los valores de TAN y azufre total del crudo, se realizó por dilución con aceite mineral blanco. Para aumentar el valor de TAN se adicionó ácido nafténico sintético de grado analítico y para modificar el valor de azufre total de agregó el compuesto dimetil disulfuro.

\subsection{Pruebas experimentales}

Las pruebas experimentales de inmersión se llevaron a cabo en un autoclave dinámico, de referencia Parr 4848 de $1800 \mathrm{ml}$ de capacidad, con una temperatura máxima de trabajo de $500^{\circ} \mathrm{C}$ y una presión máxima de $34.47 \times 10^{6} \mathrm{~Pa}$. Las pruebas realizadas se determinaron teniendo en consideración un diseño de experimentos (DE), con el fin de establecer la influencia de las principales variables en la velocidad de corrosión del material, dichos valores mencionados para cada una de las variables se escogieron acorde a los datos obtenidos tanto en un pozo productor en un campo de extracción en Colombia y la caracterización del crudo (Ver tabla 2). 
Tabla 2: Condiciones de las pruebas realizadas.

\begin{tabular}{|c|c|c|c|c|c|}
\hline Pruebas & $\begin{array}{c}\text { Temperatura } \\
{\left[{ }^{\circ} \mathrm{C}\right]}\end{array}$ & $\begin{array}{c}\text { Presión } \\
{[\mathrm{Pa}]}\end{array}$ & $\begin{array}{c}\text { Revoluciones } \\
\text { [Rpm] }\end{array}$ & $\begin{array}{c}\text { TAN } \\
\text { [g KOH/g crudo] }\end{array}$ & $\begin{array}{c}\text { Azufre } \\
\text { [\%peso total] }\end{array}$ \\
\hline $\mathrm{P} 1$ & \multirow{8}{*}{190} & \multirow{4}{*}{$8.96 \times 10^{6}$} & \multirow{2}{*}{50} & 6,84 & 0,5 \\
\hline $\mathrm{P} 2$ & & & & 2,28 & 1,5 \\
\hline P3 & & & \multirow{2}{*}{150} & 2,28 & 0,5 \\
\hline $\mathrm{P} 4$ & & & & 6,84 & 1,5 \\
\hline P5 & & \multirow{4}{*}{$11.72 \times 10^{6}$} & \multirow{2}{*}{50} & 2,28 & 0,5 \\
\hline P6 & & & & 6,84 & 1,5 \\
\hline P7 & & & \multirow{2}{*}{150} & 6,84 & 0,5 \\
\hline P8 & & & & 2,28 & 1,5 \\
\hline P9 & 220 & 1500 & 100 & 4,56 & 1 \\
\hline $\mathrm{P} 10$ & \multirow{8}{*}{250} & \multirow{4}{*}{$8.96 \times 10^{6}$} & \multirow{2}{*}{50} & 2,28 & 0,5 \\
\hline P11 & & & & 6,84 & 1,5 \\
\hline P12 & & & \multirow{2}{*}{150} & 2,28 & 1,5 \\
\hline $\mathrm{P} 13$ & & & & 6,84 & 0,5 \\
\hline P14 & & \multirow{4}{*}{$11.72 \times 10^{6}$} & \multirow{2}{*}{50} & 2,28 & 1,5 \\
\hline P15 & & & & 6,84 & 0,5 \\
\hline P16 & & & \multirow{2}{*}{150} & 2,28 & 0,5 \\
\hline P17 & & & & 6,84 & 1,5 \\
\hline
\end{tabular}

Las pruebas de espectroscopia de impedancia electroquímica (EIS) se llevaron a cabo con un montaje de vidrio [6] (Ver Figura 1), en donde se usó un potenciostato/galvanostato Gamry 600 y se utilizaron como electrodos de referencia y auxiliar un electrodo de $\mathrm{Ag} / \mathrm{AgCl}$ para altas temperaturas y un electrodo de acero inoxidable AISI/SAE 316L respectivamente, y como electrodo de trabajo los cupones del acero P110.

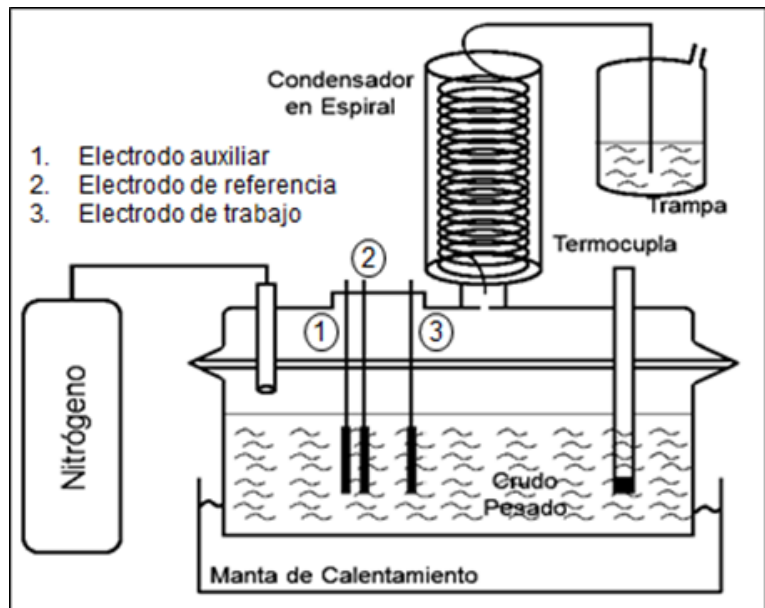

Figura 1: Montaje en vidrio para las pruebas electroquímicas

Las pruebas de EIS se realizaron con un barrido de frecuencias desde $100000 \mathrm{~Hz}$ hasta una frecuencia final de $0.01 \mathrm{~Hz}$ a una amplitud de $10 \mathrm{mV}$.

\subsection{Caracterización de los productos de corrosión}

El análisis de los productos de corrosión y la identificación del daño causado por las condiciones experimentales se realizaron en microscopio electrónico de barrido (SEM) de referencia Quanta FEG 650 y por difracción de rayos-X (DRX) en un equipo de referencia Bruker, modelo D8 DISCOVER. 


\section{RESULTADOS}

\subsection{Velocidad de corrosión del acero API P110}

Para la evaluación de la velocidad de corrosión del acero API P110, se realizaron los cálculos teniendo en consideración la norma ASTM G1(2011). Con base en lo anterior, en la tabla 3 se pueden ver los valores de la velocidad de corrosión, en mpy, obtenidos en cada uno de los ensayos propuestos.

Tabla 3: Velocidades de corrosión obtenidas en cada una de las pruebas realizadas

\begin{tabular}{|c|c|c|c|c|c|c|}
\hline \multirow{3}{*}{ PRUEBA } & \multicolumn{5}{|c|}{ CONDICIONES DE CADA PRUEBA } & \multirow{3}{*}{$\begin{array}{c}\text { V }_{\text {corr }} \text { Promedio } \\
{[\text { Mpy }]}\end{array}$} \\
\hline & Temperatura & Presión & Revoluciones & TAN & Azufre & \\
\hline & {$\left[{ }^{\circ} \mathrm{C}\right]$} & [Pa] & [RPM] & g KOH/gcrudo & \%Wtotal & \\
\hline P1 & \multirow{8}{*}{190} & \multirow{4}{*}{$8.96 \times 10^{6}$} & \multirow{2}{*}{50} & 6,84 & 0,5 & 8,97 \\
\hline $\mathrm{P} 2$ & & & & 2,28 & 1,5 & 4,22 \\
\hline P3 & & & \multirow{2}{*}{150} & 2,28 & 0,5 & 5,70 \\
\hline $\mathrm{P} 4$ & & & & 6,84 & 1,5 & 42,71 \\
\hline $\mathrm{P} 5$ & & \multirow{4}{*}{$11.72 \times 10^{6}$} & \multirow{2}{*}{50} & 2,28 & 0,5 & 6,07 \\
\hline P6 & & & & 6,84 & 1,5 & 31,64 \\
\hline P7 & & & \multirow{2}{*}{150} & 6,84 & 0,5 & 5,88 \\
\hline P8 & & & & 2,28 & 1,5 & 1,53 \\
\hline P9 & 220 & 1500 & 100 & 4,56 & 1 & 9,31 \\
\hline $\mathrm{P} 10$ & \multirow{8}{*}{250} & \multirow{4}{*}{$8.96 \times 10^{6}$} & \multirow{2}{*}{50} & 2,28 & 0,5 & 1,81 \\
\hline P11 & & & & 6,84 & 1,5 & 3,85 \\
\hline $\mathrm{P} 12$ & & & \multirow{2}{*}{150} & 2,28 & 1,5 & 36,33 \\
\hline $\mathrm{P} 13$ & & & & 6,84 & 0,5 & 6,55 \\
\hline $\mathrm{P} 14$ & & \multirow{4}{*}{$11.72 \times 10^{6}$} & \multirow{2}{*}{50} & 2,28 & 1,5 & 59,92 \\
\hline $\mathrm{P} 15$ & & & & 6,84 & 0,5 & 23,21 \\
\hline P16 & & & \multirow{2}{*}{150} & 2,28 & 0,5 & 3,37 \\
\hline P17 & & & & 6,84 & 1,5 & 3,82 \\
\hline
\end{tabular}

Las condiciones en las se presentaron mayores velocidades de corrosión del acero corresponden principalmente a las pruebas en donde el material fue expuesto al crudo con altos contenidos de azufre total y TAN, siendo esta la solución más agresiva debido a que el crudo cuenta con gran cantidad de agentes corrosivos activados térmicamente, los cuales favorecen a la corrosión por $\mathrm{H}_{2} \mathrm{~S}$ y por efecto de la acidez. La influencia en la corrosión por la formación de $\mathrm{H}_{2} \mathrm{~S}$ a partir de los compuestos orgánicos de azufre del crudo, se ve favorecido desde temperaturas de alrededor de $200^{\circ} \mathrm{C}$, ya que desde esta temperatura se forma $\mathrm{H}_{2} \mathrm{~S}$ a partir de la termólisis de compuestos orgánicos formando, por reacciones químicas, FeS. A bajos contenidos de azufre (contenidos de azufre inferior al 3\% en peso) las escamas o los productos de corrosión formados, no son estables, presentando baja adherencia en la superficie y no es una capa homogénea, por lo tanto permiten el paso de los agentes agresivos o componentes ácidos del crudo, los cuales penetran en entre las escamas y reacciona con el sustrato metálico directamente, aumentando así la velocidad de corrosión [7, 8]

Acorde a los resultados obtenidos anteriormente, se realizó un análisis estadístico según el DE propuesto. En la figura 2, el diagrama de Pareto permite comparar de forma gráfica las variables independientes y de igual manera las interacciones más influyentes en el proceso corrosivo en el sistema trabajado. Se puede observar en el diagrama de Pareto que la variable independiente más influyente en el deterioro del acero P110 es el contenido de azufre total presente en el crudo, pues teniendo en cuenta el análisis estadístico, al aumentar el contenido de azufre este favorece la corrosión del acero, mientras que las demás variables independientes tienen menor relevancia. 


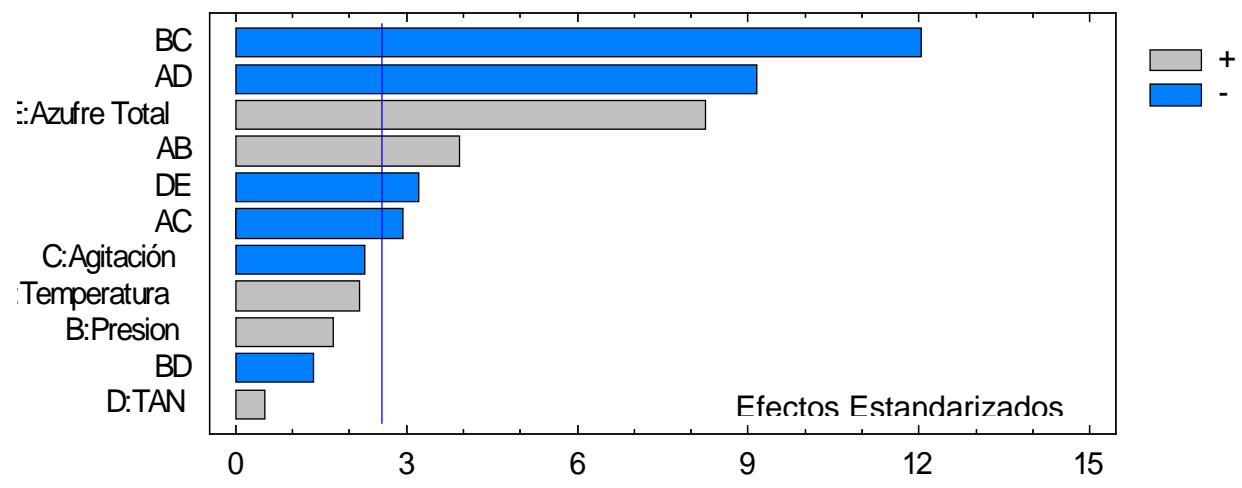

Figura 2: Diagrama de Pareto estandarizado para la velocidad de corrosión

Por el contrario, en las interacciones que se obtuvieron acorde al DE, se encontró que las más influyentes en la corrosión bajo las condiciones trabajadas, son la combinación entre la presión y la agitación, así como también la temperatura y la acidez, pues al aumentar estas interacciones la corrosión no es tan agresiva. El DE obtuvo un $\mathrm{R}^{2}$ de $99,28 \%$.

\subsection{Análisis por espectroscopía de impedancia electroquímica (EIS)}

Con ayuda de EIS se hizo un análisis de los productos de corrosión formados en la superficie del acero API P110. Inicialmente y antes de realizar las mediciones de EIS, se llevaron a cabo pruebas de potencial a circuito abierto (OCP, por sus siglas en ingles), con un tiempo de duración de 3600 segundos aproximadamente, con el fin de estabilizar el sistema e igualar las condiciones en todas las muestras ensayadas antes de llevar a cabo los ensayos de impedancia.

Para mayor comodidad y poder visualizar más fácilmente los resultados de EIS, el análisis de impedancia se realizó con los diagramas obtenidos por simulación con circuitos equivalentes a partir de los resultados experimentales. Dichas simulaciones presentaron un porcentaje de error (\%) no mayor al 10\% en cada uno de los componentes del circuito y presentaron valores de chi- squared menores a $1 \times 10^{-3}$.

En la figura 3, se pueden observar las curvas de Nyquist para la prueba P1 y la prueba P3, los cuales exhiben una diminución del diámetro de los domos capacitivos indicando una diminución de la velocidad de corrosión, debido principalmente a la diminución de la acidez del crudo. Además según el comportamiento de las curvas de Nyquist, no se evidencia la formación de una capa estable en la superficie de la muestra (ya que solo se evidencia la formación de un semicírculo). En la prueba P1, la acidez del crudo (TAN de 6,84 g $\mathrm{KOH} / \mathrm{g}_{\text {crudo }}$ ) y la temperatura trabajada ayudan a disolver los productos de corrosión formados, esto se evidencia en el lazo inductivo que se puede apreciar a bajas frecuencias, atribuido a la absorción de especies o la disolución del producto de reacción formado en la superficie metálica.

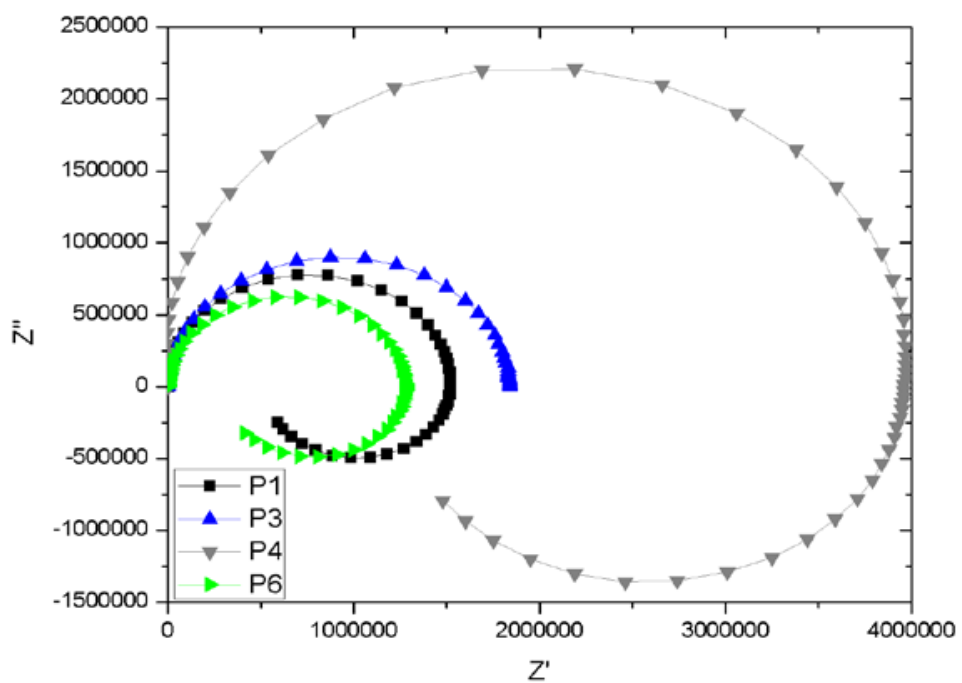

Figura 3: Curva de Nyquist: Pruebas P1, P3, P4 y P6. 
La prueba P4 y la prueba P6 presentaron altas velocidad de corrosión (determinado por gravimetría) pues el acero P110 está expuesto a la solución más agresiva, lo cual favorece el deterioro del acero, ya que la corrosión estará beneficiada por la influencia que tiene los agentes corrosivos asociados a la acidez y al azufre del crudo, presentando corrosión por ácido nafténico y corrosión por $\mathrm{H}_{2} \mathrm{~S}$. A su vez la presencia de un lazo inductivo indica la absorción de especies en el crudo, es decir, un desprendimiento y disolución de algunos productos de corrosión.

El daño superficial observado por microscopia óptica de las probetas ensayadas de las pruebas P1, P3, P4 y P6; se pueden ver en la figura 4, en la cual se puede corroborar la información obtenida en las curvas de EIS, ya que en el caso de las pruebas P1 y P3 no se observa daño significativo en la superficie de la muestra, por el contrario las probetas P4 y P6 presentan un daño superficial más representativo.

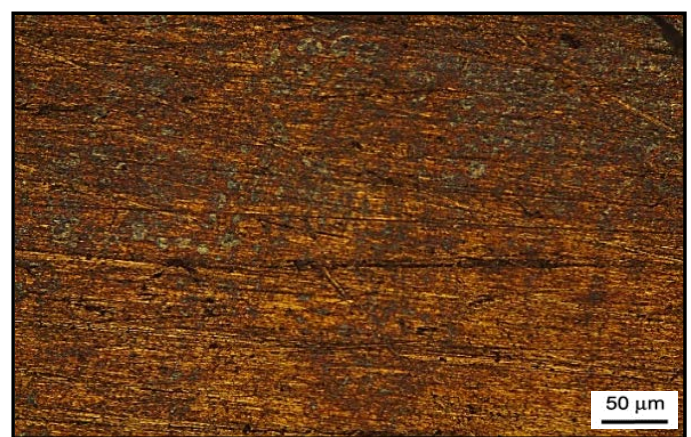

a)

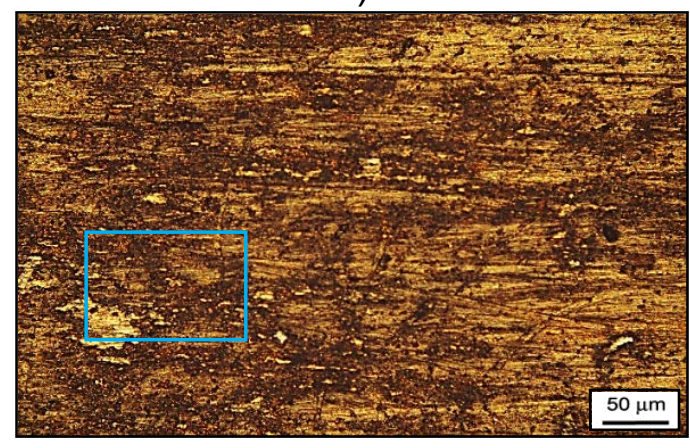

c)

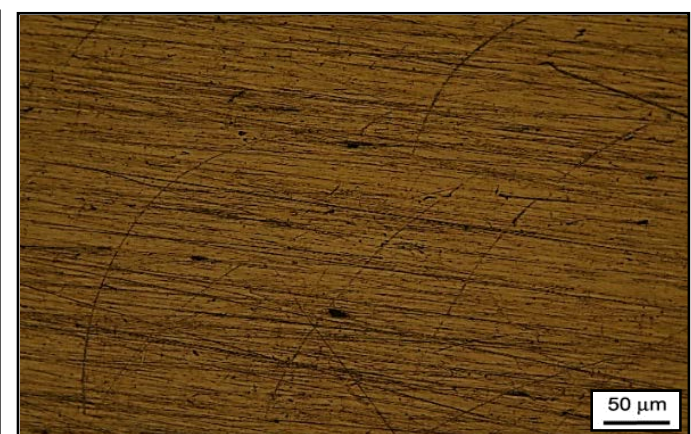

b)

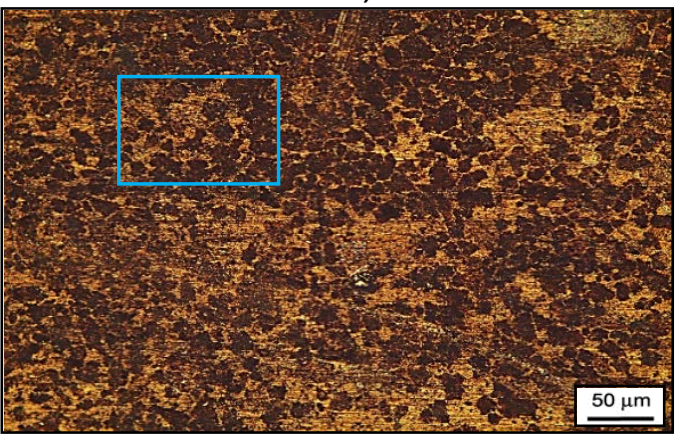

d)

Figura 4: Micrografía 10X de las pruebas: a) P1, b) P3, c) P4 y d) P6.

En la figura 5, se puede observar que a condiciones de las pruebas P2 y la P8 se presentaron velocidades de corrosión pequeñas. Como es evidente en la curva de Nyquist, existe la formación de dos domos bien definidos, lo cual se atribuye a la formación de productos de corrosión sobre la superficie metálica como consecuencia del aumento en la cantidad de azufre en el crudo, lo cual dificulta el paso de las especies agresivas del crudo a esas temperaturas y que dichas especies reaccionen con el material activo [9].

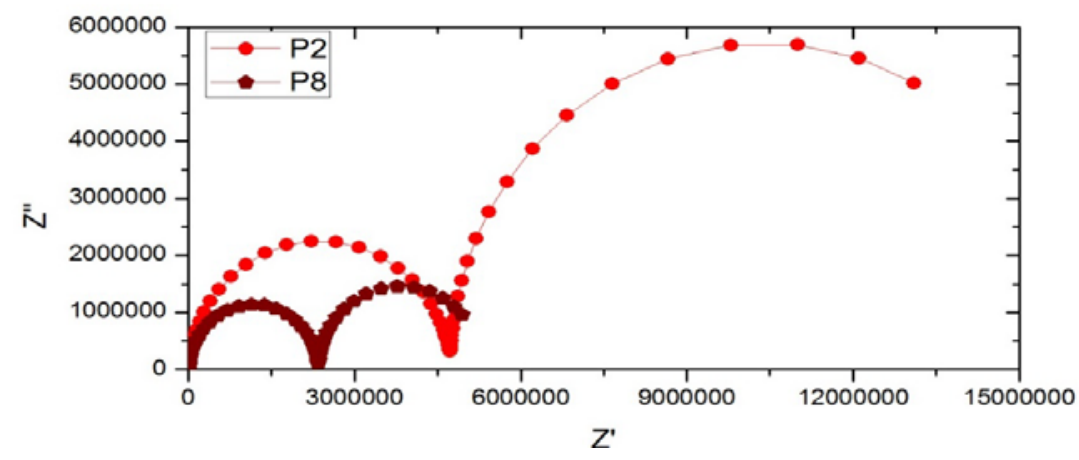

Figura 5: Curva Nyquist: Prueba P2 y P8. 
En las micrografías de las pruebas P2 y P8 (Ver figura 6) se puede observar la formación y depósito de productos de corrosión sobre la superficie metálica, los cuales se pueden atribuir al depósito de óxidos de hierro y Sulfuro de hierro, como consecuencia de las reacciones química que se dan por la influencia del azufre presente en el crudo.

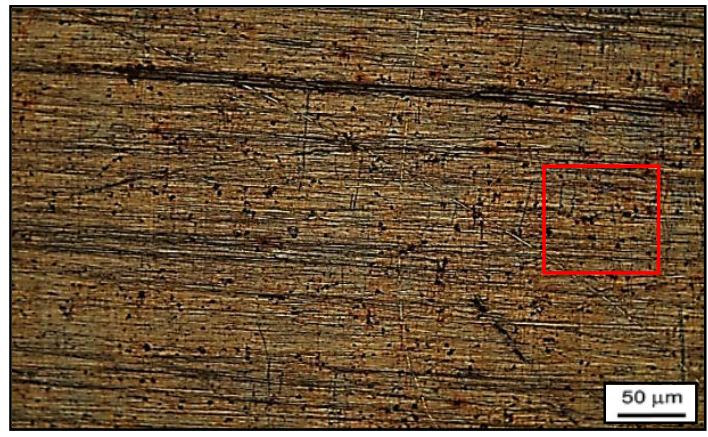

a)

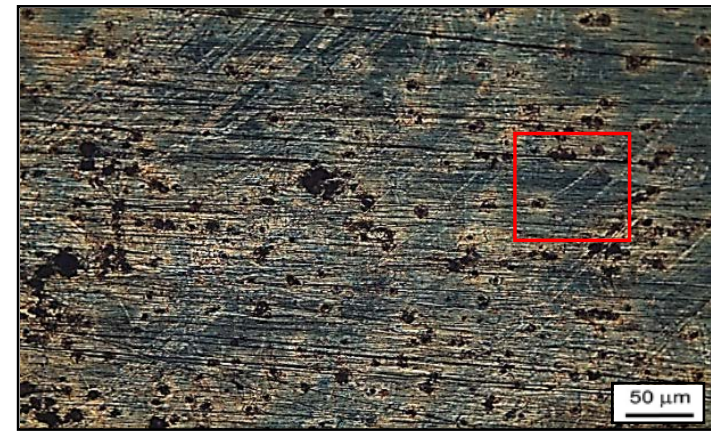

b)

Figura 6: Micrografía 10X de las pruebas: a) P2 y b) P8.

En la figura 7, los resultados obtenidos para las pruebas P11, P12 y P17 presentan un comportamiento en la curva de Nyquist muy similar.

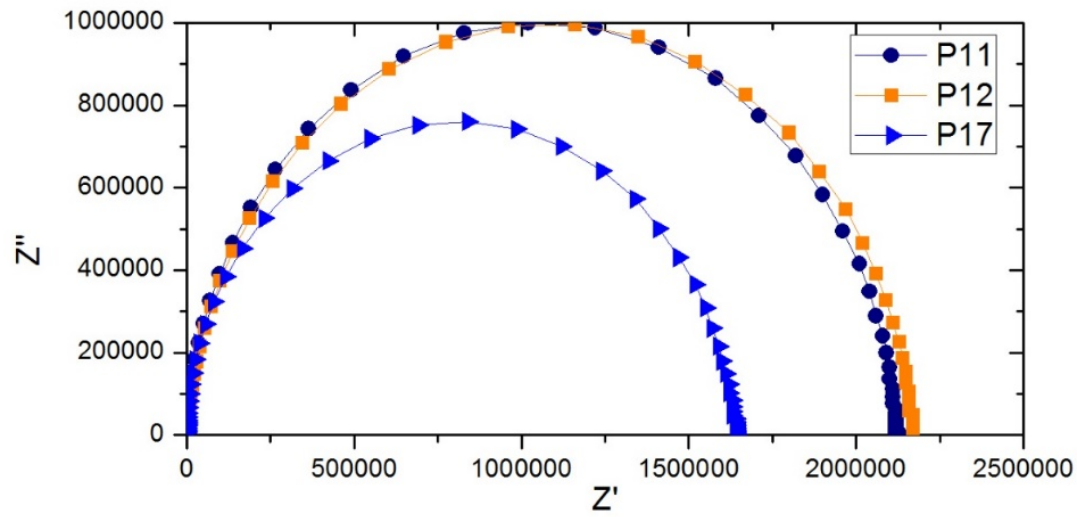

Figura 7: Curva de Nyquist: Pruebas P11, P12 y P17

Las curvas presentaron un comportamiento capacitivo de un solo domo, lo cual indica que a pesar de que se realizaron las pruebas con el crudo con alto contenido de azufre, no se presentó la formación de productos con alta estabilidad en la superficie. En el caso de las pruebas P11 y P17, existe una sinergia entre las reacciones químicas que dominan el proceso de corrosión, tanto en la formación de productos de corrosión como en la disolución por efecto de la acidez, lo cual no permite evidenciar formación alguna de una capa superficial.

Como se mencionó antes, la influencia del azufre en la corrosión es significativa en las condiciones trabajadas, como se puede ver en las figuras 8, pues se evidencia la formación de productos de corrosión por efecto del daño por $\mathrm{H}_{2} \mathrm{~S}$, pero esos productos de corrosión no disminuyen el área activa del acero, permitiendo que el crudo continúe reaccionando con este, presentando una velocidad de deterioro relativamente alta. 


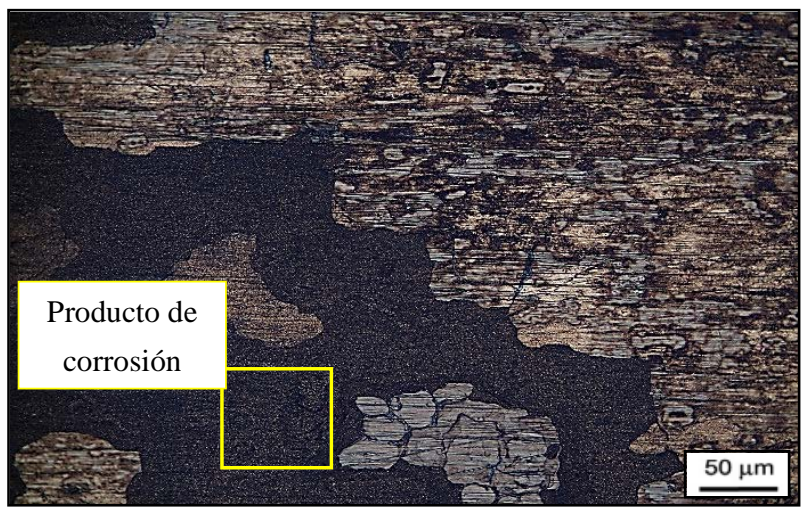

Figura 8: Micrografía 10X de la prueba: P12.

En comparación con los resultados anteriores y lo obtenido en la prueba P14 (Figura 9), la cual presentó una velocidad de corrosión elevada, se evidenció un lazo inductivo en la curva de Nyquist, asociado a reacciones químicas de reabsorción de los productos de corrosión formados en las condiciones trabajadas, en la interface FeS/Acero, pues la temperatura favorece a un aumento en la cinética de reacción por efecto de la energía termina presente. Adicionalmente, las altas presiones hacen que parte de los compuestos ácidos del crudo no cambien de estado o no se evaporen, permitiendo así una mayor cinética de reacción debido a que hay mayor tiempo de permanencia de los agentes corrosivos en el crudo.

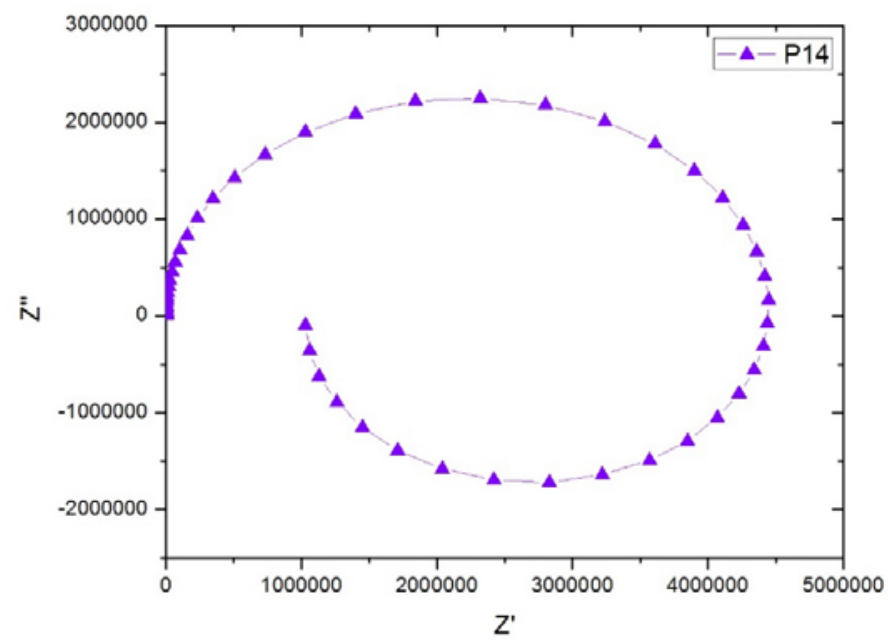

Figura 9: Curva de Nyquist: Pruebas P14

Acorde a lo observado en las curvas de EIS y por microscopia óptica (ver figura 10), se puede apreciar un gran daño superficial del acero, confirmando lo obtenido por esta técnica electroquímica.

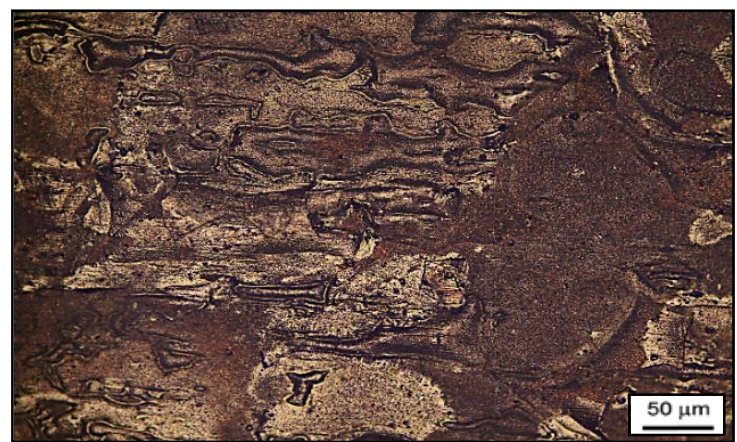

Figura 10: Micrografía 10X de la prueba: P14. 
Mediante simulaciones por circuitos equivalentes de las curvas EIS, se evaluó las propiedades eléctricas de las distintas interfaces existentes en los sistemas obtenidos en las pruebas de laboratorio, dichas interfaces pueden ser Crudo/Producto de corrosión, productos de corrosión/acero y/o Crudo/acero. Finalmente se ajustó y simularon los datos experimentales con el software Zview versión 3.1c.

Para la construcción y simulación de los diferentes circuitos eléctricos propuestos se usaron algunos componentes tales como: Resistencias (R), inductancias (L) y elementos complejos como los elementos de fase constante (CPE).

Con base en el análisis realizado anteriormente, se generaron circuitos equivalentes que representan los diagramas impedancia, tomando como base los resultados experimentales obtenidos previamente. En la figura 11, se representa el circuito equivalente propuesto que simula los resultados obtenidos para las pruebas P1 y P14. Este circuito equivalente que simuló los espectros de espectroscopia de impedancia electroquímica evidencia la formación del componente inductivo (L), asociado a la adsorción de especies en la superficie metálica por efecto de las reacciones químicas que se llevan a cabo en la doble capa electroquímica. Los valores de los componentes eléctricos que se obtuvieron en la simulación equivalen a la $\mathrm{R}_{\mathrm{sol}}$, la cual está asociada a la resistencia eléctrica que presenta el crudo a las condiciones trabajadas, el $\mathrm{CPE}_{1}$ es el equivalente a las propiedades eléctricas del acero P110, el cual hace referencia a las propiedades capacitivas y resistivas $\left(\mathrm{R}_{1}\right)$ del material base $[10,11]$.

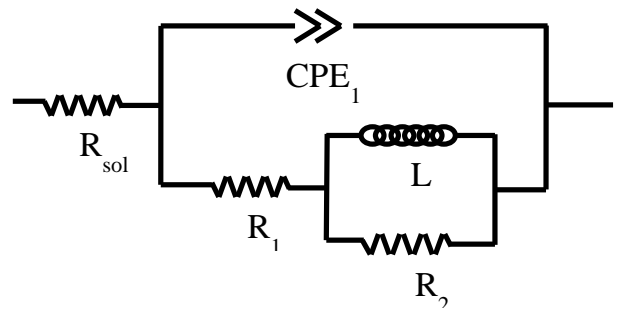

Figura 11: Circuito Equivalente I, usado para simular las pruebas P1 y P14.

El circuito de la figura 12, representa al circuito que simula las pruebas P2 y P8, este circuito a diferencia del circuito equivalente anterior, evidencia la formación de algún tipo de película formada por los productos de corrosión sobre el acero P110, por lo tanto se usó un elemento de fase adicional ( $\left.\mathrm{CPE}_{2}\right)$. Para este circuito, el $\mathrm{CPE}_{1}$ y la $\mathrm{R}_{1}$, están asociadas a las propiedades eléctricas que aportan los productos de corrosión formados y la resistencia a la trasferencia de carga que estos presentan al paso de los iones corrosivos por la capa formada. Y la $\mathrm{CPE}_{2}$ y la $\mathrm{R}_{2}$ a la capacitancia y resistencia del acero P110.

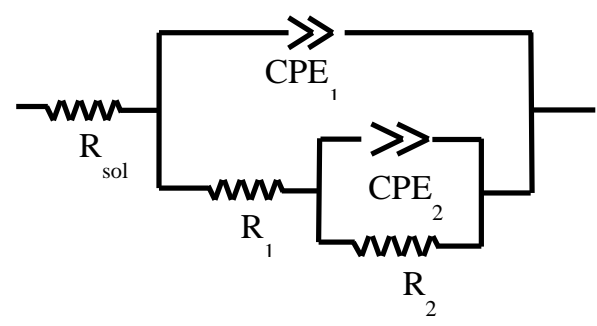

Figura 12: Circuito Equivalente II, usado para simular las pruebas P2 y P8.

En el circuito propuesto para las pruebas P12 (Ver figura 13), en el cual solo se tuvo la necesidad de utilizar un circuito simple con 3 componentes, una $\mathrm{R}_{\text {sol }}$, equivalente a la resistencia del crudo, y el $\mathrm{CPE}_{1} \mathrm{y}_{1}$, cuyos valores representan las propiedades eléctricas del acero P110 [12].

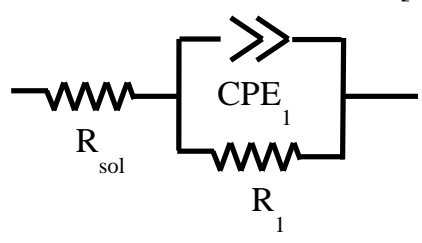

Figura 13: Circuito Equivalente III, usado para simular las pruebas P12. 
En la tabla 4, se especifica los datos simulados, en el cual se pueden observar las propiedades eléctricas obtenidas para los diferentes componentes usados en los 3 circuitos propuestos.

Tabla 4: Resultados de la simulación de los circuitos equivalentes para cada prueba

\begin{tabular}{c|c|c|c|c|c|c|c|c}
\hline \multirow{2}{*}{ Prueba } & \multirow{2}{*}{ Rsol } & \multicolumn{2}{|c|}{ CPE 1 } & \multirow{2}{*}{ R1 } & \multicolumn{2}{c}{ CPE 2 } & \multirow{2}{*}{ L } & \multirow{2}{*}{ R2 } \\
\cline { 3 - 4 } \cline { 6 - 8 } & & $\mathbf{P}$ & $\mathbf{T}$ & & $\mathbf{P}$ & $\mathbf{T}$ & & \\
\hline P1 & $1,04 \mathrm{E}+03$ & $5,98 \mathrm{E}-11$ & 0,842 & $5,23 \mathrm{E}+05$ & --- & --- & $2,93 \mathrm{E}+05$ & $9,96 \mathrm{E}+05$ \\
\hline P2 & $2,06 \mathrm{E}-04$ & $8,18 \mathrm{E}-11$ & 0,792 & $4,74 \mathrm{E}+06$ & $5,79 \mathrm{E}-08$ & 0,83 & --- & $1,13 \mathrm{E}+07$ \\
\hline P8 & $2,76 \mathrm{E}+03$ & $9,25 \mathrm{E}-11$ & 0,809 & $2,34 \mathrm{E}+06$ & $1,38 \mathrm{E}-07$ & 0,82 & --- & $2,96 \mathrm{E}+06$ \\
\hline P11 & $1,03 \mathrm{E}-01$ & $5,97 \mathrm{E}-11$ & 0,864 & $5,70 \mathrm{E}+05$ & $2,97 \mathrm{E}-10$ & 0,63 & --- & $1,56 \mathrm{E}+06$ \\
\hline P12 & $4,28 \mathrm{E}-02$ & $1,50 \mathrm{E}-10$ & 0,772 & $2,17 \mathrm{E}+06$ & --- & --- & --- & -- \\
\hline P14 & $6,32 \mathrm{E}+02$ & $1,38 \mathrm{E}-10$ & 0,827 & $1,02 \mathrm{E}+06$ & --- & --- & $7,82 \mathrm{E}+04$ & $3,43 \mathrm{E}+06$ \\
\hline
\end{tabular}

Se puede observar que todos los valores del $\mathrm{CPE}_{1}$-T están en un rango entre 0.5 y 1 , el cual describe la distribución dieléctrica de la doble capa electroquímica a las frecuencias trabajadas. De acuerdo a otros trabajos de investigación cuando los valores de $\mathrm{CPE}_{1}$-T tienen valores iguales a 0.5 es considerado impedancia de warburg, es decir un control del proceso por difusión, mientras que cuando dicho valor es igual a 1 se considera como un capacitor ideal, por lo tanto el control de todos estos procesos analizados puede estar dado por un control mixto. Para el caso de las pruebas cuyo circuito es el circuito II, indica que el comportamiento eléctrico de la capa de sulfuro de hierro puede ser pasivo, dificultando el paso de los compuestos corrosivos presentes en el crudo que puedan relacionar con el material activo, pero que debido a las condiciones trabajadas, presentan desprendimiento de grandes áreas superficiales, por lo tanto no presentan un bueno comportamiento protector $[6,13]$.

\subsection{Caracterización de los productos de corrosión}

En las figuras 14 y 15, se presenta una imagen SEM de la superficie de la muestra de acero P110 obtenida en condiciones de la prueba P4, en la cual se puede observar una superficie lisa, constituida principalmente por hierro y la presencia de algunos óxidos que no ejercen una influencia representativa en la mitigación de la velocidad de corrosión, pues en la prueba P4, se presenta una alta velocidad de corrosión, pero debido a que el acero está expuesto a las condiciones más agresivas del crudo cualquier producto de corrosión formado, se disuelve y es removido de la superficie del material. Por lo anterior no se evidencia la formación de una capa pasiva o protectora sobre la muestra.

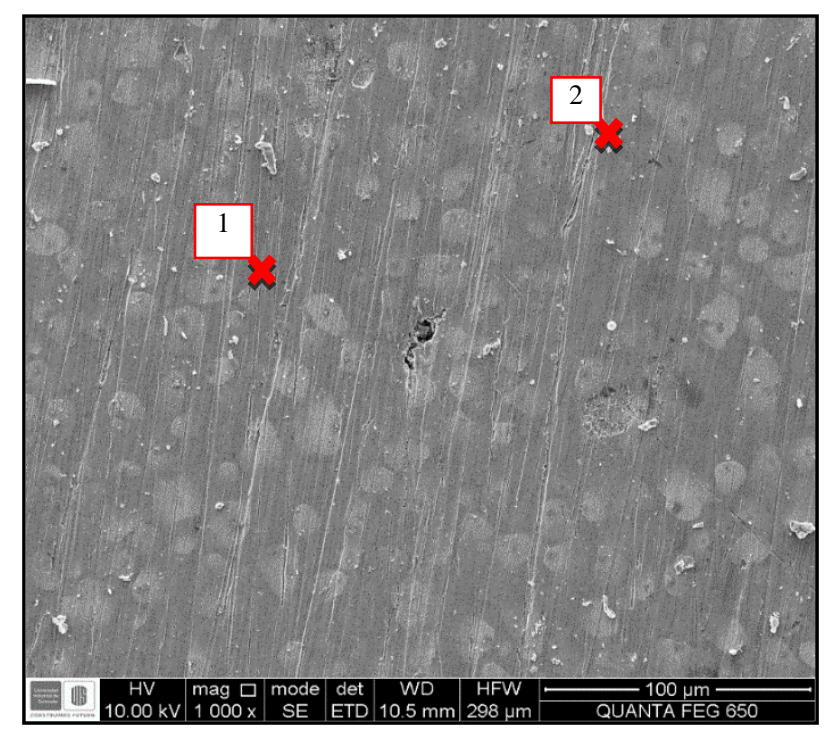

Figura 14: SEM Prueba P4 


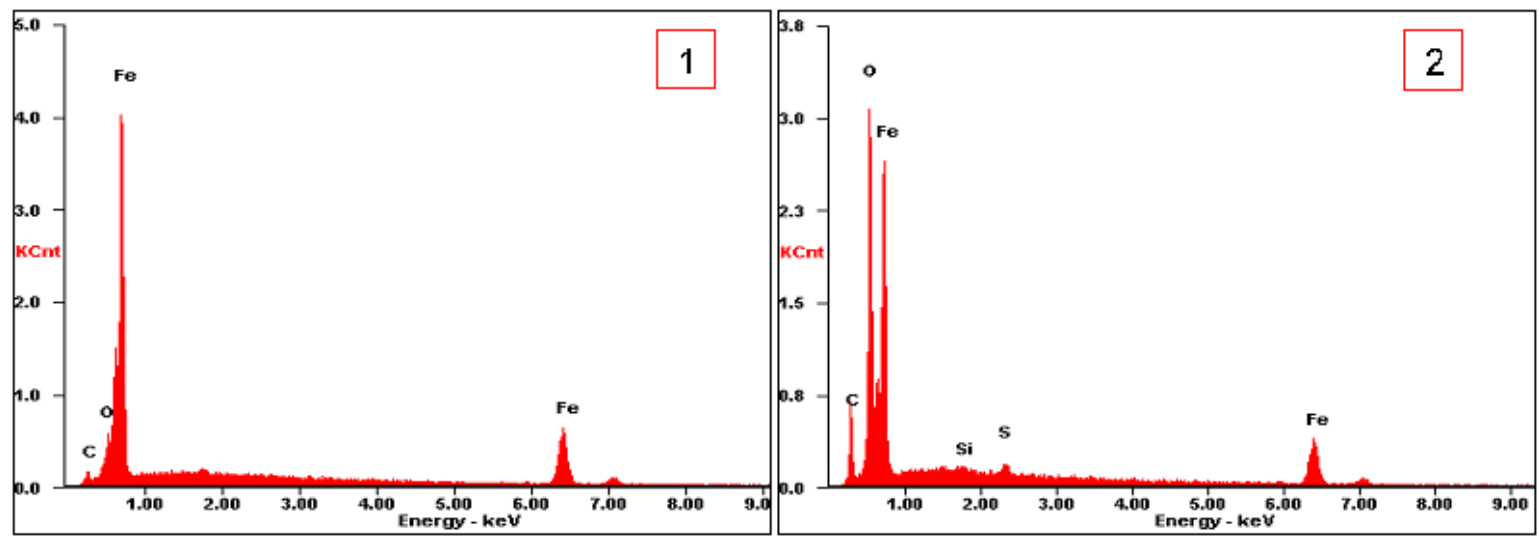

Figura 15: EDS Prueba P4

Para el caso de la prueba P14, en la cual se presento la mayor velocidad de corrosión (Ver figura 16 y 17), se evidencia la presencia de escamas sobre la superficie del material, formadas principalmente como consecuencia de las reacciones químicas que se llevan a cabo en la corrosión por $\mathrm{H}_{2} \mathrm{~S}$, generando compuestos con alto contenido de Fe y S, asociados con la formación de FeS. Estos productos de corrosión formados no presentan propiedades protectoras, pues las condiciones usadas para los experimentos, es decir, condiciones que se acerquen al procesamiento de crudo, no favorece a la formación de capas estables pasivas que permitan disminuir la velocidad de corrosión, por el contrario favorece la corrosión por efecto del azufre del crudo como principal mecanismo de deterioro del acero [14].

En las figuras 18, se puede apreciar el espesor que pueden tener dichas escamas y su respectivo analisis EDS, los cuales pueden estar alrededor de $1.5 \mu \mathrm{m}$ de espesor, presentando escamas discontinuas y de manera heterogenea sobre la superficie metalica, presentando un comportamiento no pasivo por la gran discontinuidad que esta presenta.

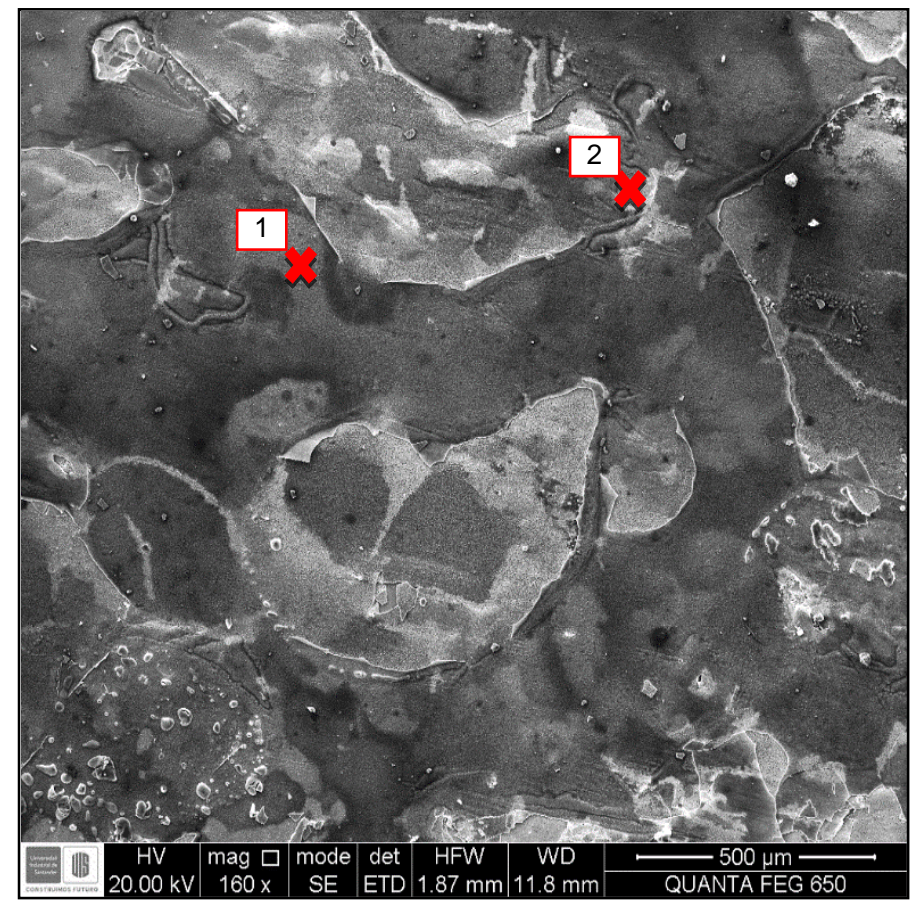

Figura 16: SEM Prueba P14 

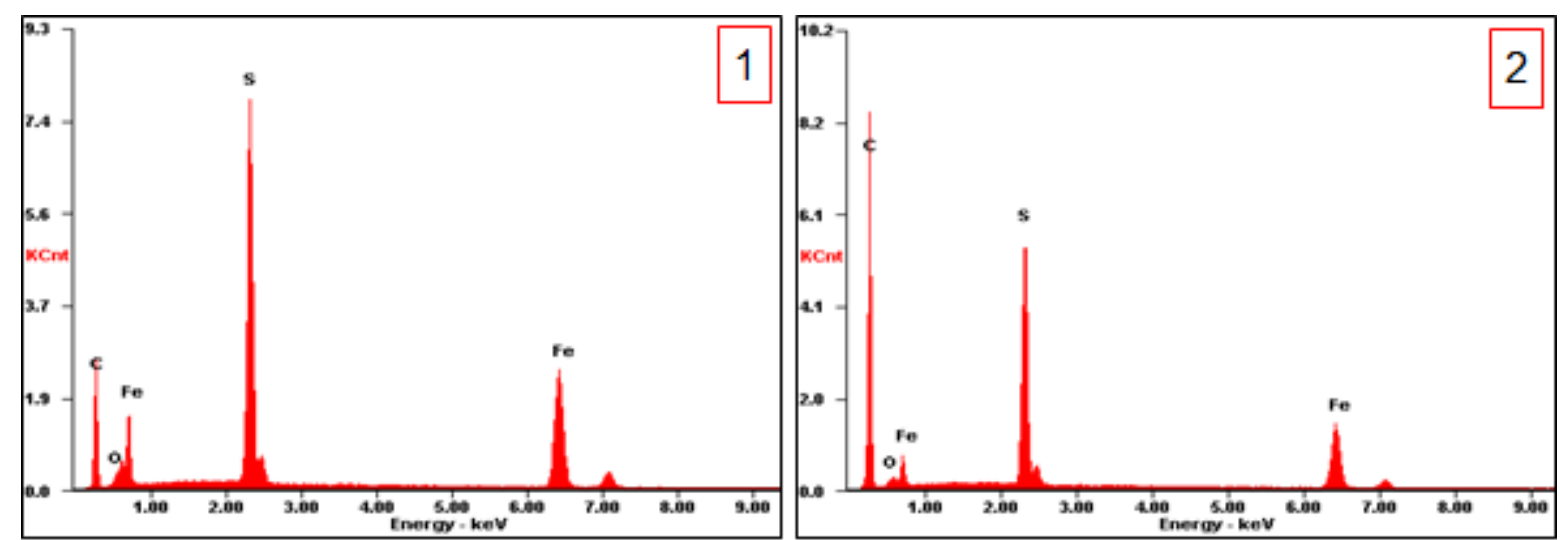

Figura 17: EDS Prueba P14

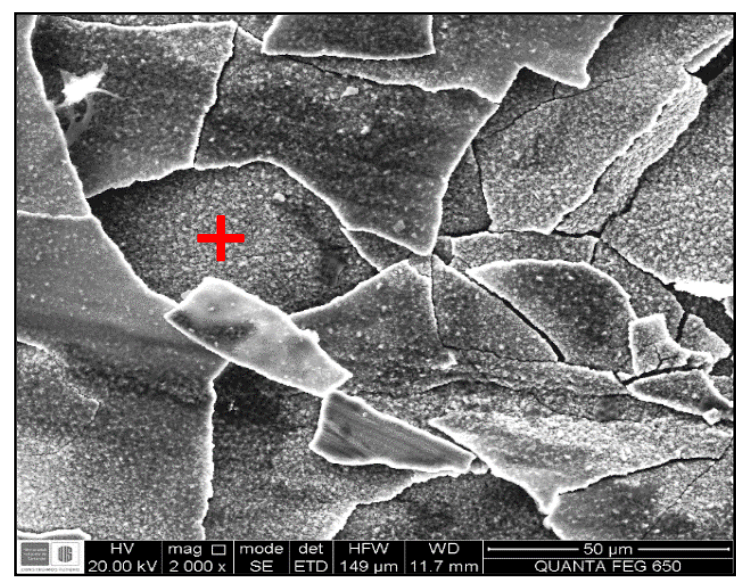

a)

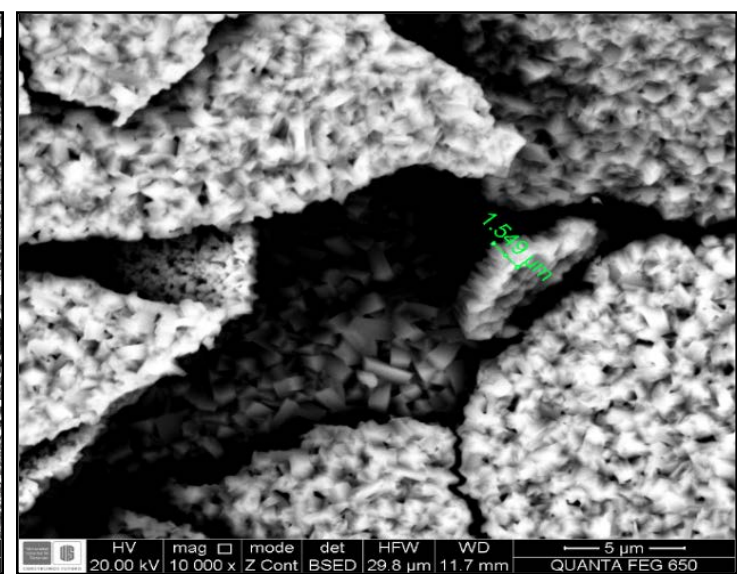

b)

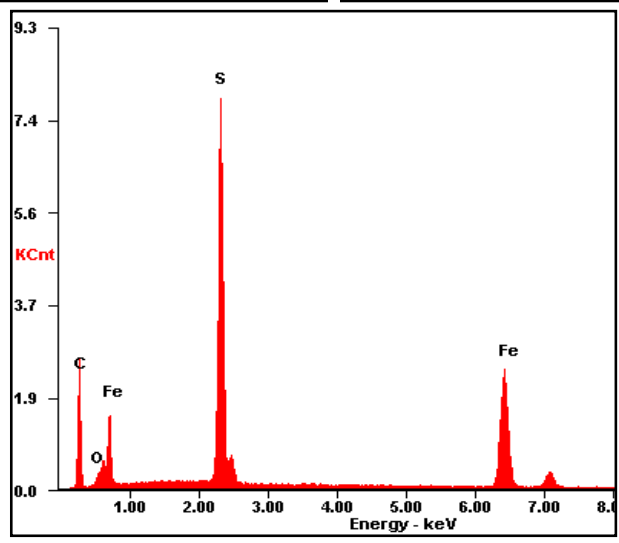

C)

Figura 18: SEM de la probeta P14. a) A $50 \mu \mathrm{m}$, b) a $5 \mu \mathrm{m}$ de aumento y c) EDS de los productos formados.

Por análisis por difracción de rayos $\mathrm{X}$, se encontró que los productos de corrosión formados sobre la superficie metálica fueron: Pirrotita 7T (Fe1-xS), óxido de hierro $\left(\mathrm{Fe}_{3} \mathrm{O}_{4}\right)$, Sulfuro y hierro $\left(\mathrm{Fe}_{0.975} \mathrm{~S}\right) \mathrm{Y}$ hierro (Fe). La formación de FeS está dada principalmente a las reacciones químicas que se llevan a cabo en el mecanismo de corrosión debido a la formación de $\mathrm{H}_{2} \mathrm{~S}$, siendo este el principal mecanismo de deterioro el cual controla los procesos corrosivos bajo las condiciones ensayadas.

Acorde a los resultados obtenidos por gravimetría se estableció que de las variables independientes trabajadas la única que tiene relevancia y gran influencia en el proceso corrosivo del acero API P110 es el contenido de azufre total del crudo, pues debido a la presencia de estos compuestos químicos se promueve la formación de productos de reacción sobre la superficie metálica, como se pudo observar por análisis de difracción de rayos x, respecto a la formación de sulfuro de hierro (FeS). Adicionalmente, el análisis estadístico realizado estableció que la variación de la temperatura, presión y agitación, no representan gran influencia en el deterioro del acero en comparación con la variación del contenido de azufre, pero cabe resaltar que a estas 
condiciones de temperatura se ven favorecidos los mecanismos de corrosión tanto por ácido nafténico y $\mathrm{H}_{2} \mathrm{~S}$.

Mediante el análisis realizado para las pruebas ensayadas a elevadas presiones y temperaturas por EIS, se presentaron comportamientos que permiten suponer y proponer un posible mecanismo de deterioro del acero influenciado por las condiciones de laboratorio trabajadas (Ver figura 19).

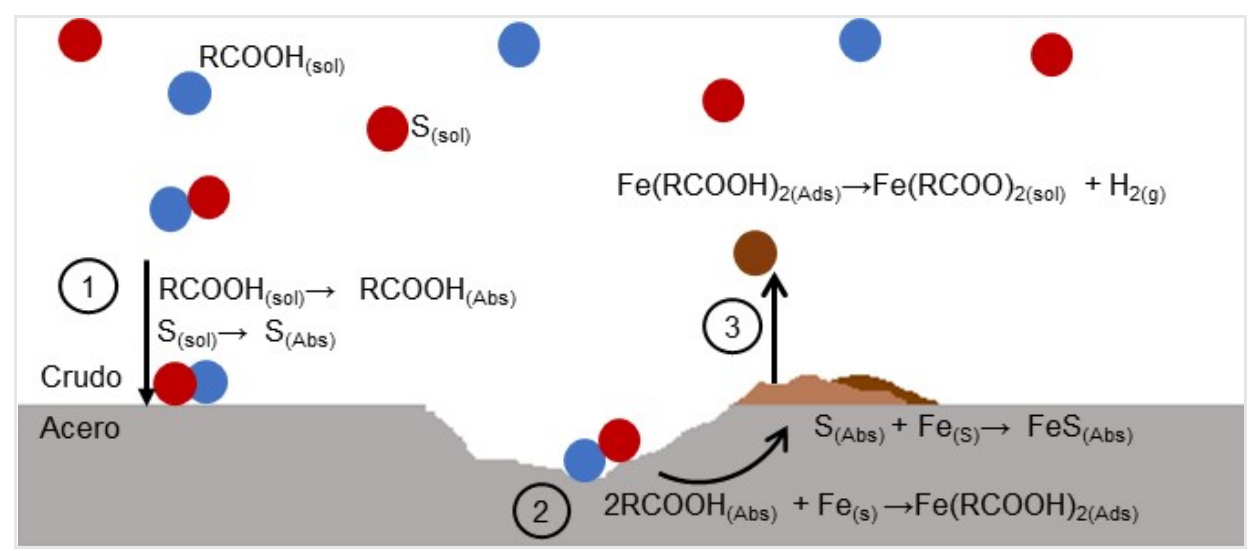

Figura 19: Posible mecanismo de corrosión a las condiciones trabajadas.

Inicialmente las reacciones químicas que se pueden dar en la superficie del acero están determinadas por la difusión de las especies agresivas solubles en el crudo, haciendo énfasis en los compuestos de ácido nafténico y compuestos de azufre activados térmicamente, dadas las condiciones de agitación trabajadas las cuales promueven la absorción de dichos compuestos en la superficie metálica.

$\mathrm{RCOOH}_{\text {(soluble) }}+\mathrm{S}_{\text {(compuestos)(soluble) }} \rightarrow \mathrm{RCOOH}_{(\text {Abs) }}+\mathrm{S}_{\text {(compuestos)( (Abs) }}$

Estos compuestos absorbidos en la doble capa electroquímica pueden reaccionar con el hierro presente en el material, como se evidenció en las curvas de Nyquist en algunas de las pruebas ensayadas, en las que se presentó dos domos capacitivos o dos constantes de tiempo en las curvas de bode: frecuencia vs IZI, las cuales se atribuyen a las reacciones químicas dando lugar a los procesos de corrosivos, que de acuerdo con el análisis del DE, está dado principalmente por el mecanismo de corrosión por efecto de azufre, dando como resultado las siguientes posibles reacciones $[7,15,16]$ :

$$
\begin{aligned}
& 2 \mathrm{RCOOH}_{(\mathrm{Abs})}+\mathrm{Fe}_{(\mathrm{S})} \rightarrow{\mathrm{Fe} \mathrm{RCOOH})_{2(\mathrm{Ads})}}+\mathrm{ReS}_{\text {(compuestos (Abs) }_{(\mathrm{Abs})}}+\mathrm{Fe}_{(\mathrm{S})} \rightarrow \mathrm{FeS}_{(\mathrm{Abs})}
\end{aligned}
$$

Finalmente, y de acuerdo con los resultados obtenidos por EIS, se evidencia también la influencia de procesos de absorción y de redisolución de productos de corrosión formados, las cuales se pueden evidenciar en las curvas de Nyquist en donde a bajas frecuencias se pueden apreciar lazos inductivos, pues diversos autores la asocian a este fenómeno de disolución. [7, 15, 16, 17, 18, 19].

$\mathrm{Fe}(\mathrm{RCOOH})_{2(\mathrm{Ads})} \rightarrow \mathrm{Fe}(\mathrm{RCOO})_{2(\mathrm{sol})}+\mathrm{H}_{2(\mathrm{~g})}$

Teniendo en consideración los resultados obtenidos y el análisis realizado, se pudo establecer que los productos de corrosión formados a condiciones de altas temperaturas y presiones, en presencia de un crudo pesado con altos contenidos de azufre total y TAN no presentan propiedades pasivas que pueden ayudar a disminuir la velocidad de corrosión, por el contrario a las condiciones trabajas se favorece la disolución y absorción de dichos productos en el crudo. 


\section{CONCLUSIONES}

El acero API P110 presento altos niveles de deterioro correspondiente a las prueba en la que se usó la solución con altos contenidos de azufre total y altos valores de TAN, siendo esta la solución más agresiva, ya que cuenta con mayor concentración de agentes corrosivos que afectan el material, pues a las temperaturas trabajadas, se favorecen la activación de mecanismos de corrosión por formación de $\mathrm{H}_{2} \mathrm{~S}$ y corrosión nafténica principalmente.

Por medio de espectroscopia de impedancia electroquímica, se pudo establecer que en la mayoría de las pruebas realizadas se presentó un lazo inductivo en las curvas de Nyquist a bajas frecuencias, la cual se atribuye a procesos de absorción de los productos formados en las reacciones químicas, favoreciendo la disolución de los productos de corrosión ayudando así al desprendimiento de dichos productos y acelerando el deterioro del material. Además los circuitos propuestos para la simulación de los diferentes resultados de espectroscopia de impedancia electroquímica, se ajustaron muy bien a los resultados experimentales obtenidos en los diferentes ensayos, presentando errores relativamente pequeños, lo cual asegura la existencia de las propiedades capacitivas de la doble capa electroquímica, así como el fenómeno de absorción de especies en la interfase metal/electrolito a bajas frecuencias.

Se determinó por medio de FESEM y DRX que los principales productos de corrosión formados fueron sulfuro de hierro y óxido de hierro, originados principalmente por las reacciones químicas que se dan en el proceso predominante de corrosión, el cual es fue la corrosión por azufre.

Teniendo en cuenta las condiciones ensayadas, es decir, las pruebas a elevadas temperaturas y altas presiones, no se favorece la formación de productos de corrosión sobre la superficie del acero, que sirvan como capas pasivas, las cuales tienen como función disminuir la velocidad de corrosión, pues bajo estas condiciones se ve favorecido la disolución y absorción de dichos productos en el crudo.

\section{AGRADECIMIENTOS}

Al grupo de investigaciones en corrosión (GIC) de la Universidad Industrial de Santander y al Instituto Colombiano del Petróleo (ICP) y Colciencias por la capacitación y colaboración en el desarrollo del trabajo. Al Centro de Materiales y Nanomateriales CMN-UIS.

A todos los profesores y técnicos en general, por su apoyo, paciencia, comprensión, asistencia y conocimiento aportado durante toda la carrera.

\section{BIBLIOGRAFÍA}

[1] DEYAB, M., ABO DIEF, H., EISSA, E. A., et al., "Electrochemical investigations of napthenic acid corrosion for carbon steel and the inhibitive effect by some ethoxilated fatty acids", Electrochemica acta, v.52, n.28, pp. 8105-8110, 2007.

[2] BOTA, G. M., DINGRONG, Q., SRDJAN, N. "Naphthenic acid corrosion of mild steel in the presence of sulfide scales formed in crude oil fractions at high temperature”, NACE, n. 10353, pp. 1-9, 2010.

[3] WEI, S., SRDJAN, N. “A mechanistic model of $\mathrm{H}_{2} \mathrm{~S}$ corrosion of mild Stee”, NACE, n. 07655, 2007.

[4] WEI, S., ANDRES, M .,GERARDINE, B. "Theoretical Investigation of $\mathrm{H}_{2} \mathrm{~S}$ Corrosion of Mild Steel," 2000. [En línea]. Available: http://www.electrochem.org/dl/ma/203/pdfs/0249.pdf.

[5] MYTHILI, K. $\mathrm{CO}_{2}$ and $\mathrm{H}_{2} \mathrm{~S}$ corrosión in oil pipelines, Faculty of Mathematics and Natural Science, University of Stavanger, 2010.

[6] JAVIER, S., Evaluación mediante tecnica gravimetrica y electroquímicas, del efecto del azufre de un ambiente que simula el procesamiento de un crudo pesado, en la velocidad de corrosion del acero ASTM A335-P9, Bucaramanga, Universidad Industrial de Santander, [s.d].

[7] DETTMAN, D., NANA, LI, WICKRAMASINGHE, D., et al, "The influence of naphthenic acid and sulfur compound structure on global crude corrosivity under vacuum distillation conditions heather" , NACE, n. C2012-0001326, pp. 1-7, 2012.

[8] HUANG B., YIN W., SANG D., et al., "Synergy effect of naphthenic acid corrosion and sulfur corrosion in crude oil distillation unit”, El SEVIER Applied Surface Science, n. 259, pp. 664-670, 2012.

[9] RIVERA GRAU, L. M., "Evaluation of the corrosion inhibition by coco-modified imidazoline in $\mathrm{CO}_{2}$ $\mathrm{H}_{2} \mathrm{~S}$ ”, NACE corrosion conference and expo, n. C2012-0001700, pp. 1-15, 2012.

[10] QUEJ-AKÉ, L., CABRERA-SIERRA, R., ARCE-ESTRADA, E., et al., "EIS Evaluation of the effect of neutralizing and inhibitor compounds on corrosion process of 1018 carbon steel in acid solutions typical of 
atmospheric distillation plants”, Internationa Journal of Electrochemical Science, v. 3, n.1, pp. 56 - 66, 2008. [11] LEE, K.L., SRDJAN, N. "The effect of trance amoint of $\mathrm{H}_{2} \mathrm{~S}$ on $\mathrm{CO}_{2}$ corrosion investigated by using the EIS technique”, Corrosion, n. 05630, pp. 1-16, 2005.

[12] LEE, K.L., Srdjan , N. "Use and abuse of EIS in studying the mechanisms of $\mathrm{CO}_{2} / \mathrm{H}_{2} \mathrm{~S}$ corrosion of mild steel," Corrosion, no 06417, pp. 1 - 30, 2006.

[13] DING, J., LU, M., GAO, C., et al., "Effect of pH on the electrochemical behavior of UNS S31803 duplex stainless steel in $\mathrm{H}_{2} \mathrm{~S}-\mathrm{CO}_{2}$-Cl- Environment," NACE Corrosion conference and Expo, n. 2526, pp. 1 9, 2013.

[14] BRAVO MENDEZ, J., DOMÍNGUEZ, M. A., GONZALES J. L., "The effect of napthenic and sulfidic corrosion on the microstructure of a steel A106-B using vacuum residue”, Mexico D.F., 2014.

[15] FREITAS, S., MALACARNE, M., ROMAO, W., et al., "Analysis of the heavy oil distillation cuts corrosion by electrospray ionization FT-ICR mass spectrometry, electrochemical impedance spectroscopy, and scanning electron microscopy”, Fuel, v. 104, pp. 656-663, 2013.

[16] ALVISI, P.P., LINS,V.F.C.“An Overview of naphthenic acid corrosión in vaccum destillation”, Engineering Failure Analysis, v. 18, n.5, pp. 1403-1406, 2011.

[17] DAVOODI, A., BABAIEE, M. Y., EBRAHIMI G., "A comparative $\mathrm{H}_{2} \mathrm{~S}$ corrosion study of 304L and 316L stainless steels in acid media”, Corrosion Science, v. 53, n.1, pp. 399-408, 2011.

[18] KANEE R. D., CAYARD, M. S., “A comprehensive study on naphthenic acid corrosion”, Corrosion, n. 02555, pp. 1-15, 2010.

[19] LI, N., Laboratory study of high temperature corrosion in petroleum refineries., Tesis de D.Sc.,University of Alberta, 2009. 